\section{Malnutrition bei COPD}

Zusammenfassung: In den letzten Jahren ist zunehmend die Erkenntnis gewonnen worden, dass bei COPD viele Faktoren für die beobachtete Unterernährung eine Rolle spielen. Hierbei wurde klar, dass eine komplexe Veränderung des Metabolismus bei COPD-Patienten vorliegt, die durch eine reine Ernährungstherapie wenig zu beeinflussen ist. In dem Übersichtsartikel wird anhand der aktuellen Literatur auf die Malnutrition und den veränderten Metabolismus bei COPD-Patienten eingegangen und die Ernährungsintervention bei diesen Patienten diskutiert. Unser Blickfeld bezüglich der Therapie muss sich dahin richten, dass wir bei der COPD nicht eine lokale Erkrankung des Bronchialbaumes haben, sondern eher eine systemische Erkrankung, die von der Lunge ausgeht und dort die Hauptsymptome verursacht, aber auch andere Systeme mit einbezieht. Ziel weiterer Studien muss es daher sein, die Muskelfunktion und die Veränderungen in diesem Bereich bei COPD-Patienten noch besser zu verstehen, um dann hierauf Einfluss nehmen zu können. Zukünftige Therapien sollten sich daher nicht nur auf die Behandlung der Inflammation und Dilatation der Bronchien beschränken, sondern darauf ausgerichtet sein, den veränderten Metabolismus bei COPD-Patienten zu beeinflussen, um so die Belastbarkeit der Patienten und vielleicht auch die Prognose zu verbessern.

Malnutrition in COPD: During the last years our knowledge about the malnutrition of patients with COPD has grown. Weight loss is a problem of some patients with COPD, but, as we know today by techniques of body composition measurement much more patients have reduced muscle mass. The reason for this is complex and involves many body systems. Nutritional intervention alone is not successful in many patients. We have to accept that COPD is a kind of systemic disease which does not only involve the lung. Thus, future therapies have to include not only treatment of the bronchial system.

\section{Einleitung}

Chronische Bronchitis, das Lungenemphysem sowie Mischformen aus beiden werden hauptsächlich durch Rauchen verursacht. Im angloamerikanischen und zunehmend auch im deutschen Sprachgebrauch werden die beiden Krankheits-

Pneumologie 2001; 55: 475-480

(c) Georg Thieme Verlag Stuttgart · New York ISSN 0934-8387
J. Bargon, U. Müller

Katharina Kasper Kliniken, St. Elisabethen Krankenhaus

Frankfurt/Main bilder unter dem Begriff COPD zusammengefasst. Die Inzidenz und die Prävalenz der COPD steigen. Trotz der Tatsache, dass Rauchen der Hauptrisikofaktor für das Erkranken an einer COPD ist, entwickeln nur ca. 15-20\% der Raucher diese Erkrankung. Es liegt daher nahe zu vermuten, dass genetische Faktoren einen Einfluss haben. Möglicherweise spielt auch die Ernährung, z. B. in Form von besserem antioxidativen Schutz, eine Rolle.

Unter- und Mangelernährung sind bei Patienten mit COPD sehr häufig. In der Literatur wird, abhängig auch von der Schwere der Erkrankung, eine Inzidenz zwischen 20\%-60\% angegeben $[4,19,21,24,34,38]$. Eine klare Korrelation zwischen Bodymass-Index (BMI) und Lungenfunktionsparametern konnten ebenso gefunden werden wie zwischen BMI und Mortalität [5,34].

\section{Bedeutung der Ernährung für die Atmung}

Gewichtsverlust bei Patienten mit COPD und anderen Lungenerkrankungen und die Bedeutung der Ernährung für die Atemfunktion sind seit dem Beginn des 20.Jahrhunderts mehr oder weniger bekannt. Sie haben in den letzten Jahren aufgrund neuer Erkenntnisse zunehmend an Bedeutung gewonnen. So ist der untergewichtige „pink puffer“-Emphysematiker ebenso ein alter Begriff wie der übergewichtige „blue bloater"-Patient mit chronischer Bronchitis. Diese Beschreibungen der Patienten, ebenso wie alle älteren Studien, beziehen sich nur auf das Körpergewicht. Daten über die Körperzusammensetzung dieser Patienten lagen bis vor einigen Jahren nicht vor.

Die neueren Erkenntnisse beruhen auf der Tatsache, dass man sich inzwischen mit dem viel interessanteren Anteil der Körperzusammensetzung beschäftigt, nämlich mit dem „Activity metabolizing tissue" das indirekt durch die fettfreie Masse (= Muskelmasse) gemessen werden kann. Diese ist ein viel besserer Parameter als das reine Körpergewicht. So ist inzwischen bekannt, dass auch übergewichtige COPD-Patienten oft eine niedrige Muskelmasse haben [56]. Heute weiß man, dass die Reduktion der Muskelmasse relativ früh auftritt und nicht mit dem Schweregrad der Erkrankung korreliert [42]. Diese Tatsache verlangt ein völlig neues Verständnis von dem, was wir bisher unter „pulmonaler Kachexie“ verstanden haben, die im Endstadium einer Lungenerkrankung auftritt.

Mittlerweile gibt es einige Studien, die gezeigt haben, dass der Verlust an Muskelmasse ein nicht zu vernachlässigendes Problem bei vielen COPD-Patienten darstellt. Eine erniedrigte 
Muskelmasse konnte schon bei Patienten mit nur leicht- und mittelgradiger obstruktiver Ventilationsstörung beobachtet werden. Eine Korrelation mit dem Schweregrad der Obstruktion fand sich nicht, sondern eher mit der Diffusionskapazitität $[12,53]$. Es konnte gezeigt werden, dass eine verminderte Muskelmasse besonders bei Emphysematikern zu beobachten ist: $37 \%$ der Emphysematiker und 12\% der Patienten mit chronischer Bronchitis zeigten eine erniedrigte Muskelmasse [14].

Die Beobachtung, dass bei COPD-Patienten ein Verlust an Muskelmasse vorliegt, erklärt auch die bekannte Klinik der Patienten, die unter verminderter Belastbarkeit und peripherer Muskelschwäche leiden [2,3,37,55]. Diese korrreliert deutlich mit dem Grad der Lebensqualität $[30,40]$.

\section{Folgen von Malnutrition auf die Atmung}

Der Verlust von Muskelmasse hat einen direkten Einfluss auf die Ventilation und die Atemmuskulatur. Im Gegensatz zu früheren Meinungen konnte in Studien der Achtziger Jahren gezeigt werden, dass die Atemmuskulatur ebenso wie die übrige Skelettmuskulatur den katabolen Veränderungen bei Mangelernährung und Stress in Form von reduzierter Proteinsynthese und erhöhtem Abbau unterworfen ist. Es scheint sogar so zu sein, dass der Verlust an Muskelmasse insgesamt die Atemmuskulatur prozentual mehr betrifft als die übrige Muskulatur $[1,25]$.

Außerdem führt eine Mangel- und Unterernährung zu strukturellen Veränderungen der Lunge und Atemwege sowie $\mathrm{zu}$ einer Verminderung der pulmonalen Abwehrmechanismen. In Tiermodellen wurde hierfür eine Abnahme der Makrophagen-Aktivität verantwortlich gemacht [39]. Es konnte gezeigt werden, dass Mangelernährung die antibakterielle Abwehr der Lunge vermindert [28]. Zudem kommt es zu einer Veränderung des Arachidonsäuremetabolismus in Alveolarmakrophagen mit verminderter Produktion von Leukotrien $B_{4}$ und erhöhter Freisetzung von Thromboxan $\mathrm{B}_{2}$ und Prostaglan$\operatorname{din} E_{2}[41]$.

So existiert ein oft zu wenig beachteter Circulus vitiosus: eine chronische Lungenerkrankung wie COPD führt häufig zu Malnutrition, in deren Verlauf es zu Unter- und Mangelernährung mit einer Progression der chronischen Lungenerkrankung kommt.

\section{Mechanismen der Malnutrition bei COPD}

Unter dem Begriff der pulmonalen Kachexie ist bekannt, dass Patienten im Endstadium ihrer Lungenerkrankung Gewicht verlieren. Die meisten Studien hierzu wurden bei COPDPatienten durchgeführt. Wie oben erwähnt, beginnt dieser Prozess aber bereits viel früher, nämlich bevor Untergewicht oder Kachexie zu beobachten sind, indem es zu einem Verlust der Muskelmasse kommt.

Die Ursachen hierfür sind vielfältig und noch nicht gänzlich geklärt. Eine negative Energiebilanz, die bei Gewichtsverlust bei chronisch Lungenkranken vorhanden sein muss, kann folgende Ursachen haben: Reduzierte Aufnahme von Nahrung, verminderte Absorption, erhöhter Energieverbrauch, veränderter Metabolismus.

\section{Reduzierte Aufnahme von Nahrung}

Für die verminderte Aufnahme von Nahrung bei Patienten mit fortgeschrittener Lungenerkrankung gibt es selten nur einen einzigen Grund. Es sind mehrere Faktoren, die dazu beitragen, wie

- chronische Mundatmung bei respiratorischer Insuffizienz führt zu vermindertem Geschmacksempfinden und daraus resultierendem mangelnden Interesse an Essen.

- Dyspnoe, die oft bereits in Ruhe vorhanden ist, verstärkt sich bei Belastung, z.B. auch durch Nahrungsaufnahme. Zunehmende Dyspnoe fördert Müdigkeit und Abgeschlagenheit, was zu verkürzten Essenszeiten führt. Zudem führt Dyspnoe auch dazu, dass die Patienten sich nicht selbst etwas kochen und nicht zum Essen ausgehen.

- Aerophagie, die bei COPD-Patienten häufiger ist als bei Gesunden, führt zu vorzeitigem Sättigkeitsgefühl oder sogar Beschwerden.

- Sauerstoffabhängigkeit: Gerade Patienten, die Sauerstoff über eine Maske zu sich nehmen, haben beim Essen nur die Wahl zwischen Nahrungsaufnahme oder Sauerstoff.

- Medikamente wie Antibiotika, Theophyllin, etc. führen nicht selten zu gastrointestinalen Beschwerden, die wiederum zu mangelndem Appetit und ungenügender Nahrungsaufnahme führen. Auch die Menge an Medikamenten, die oft vor oder zum Essen genommen werden sollen, führt bei manchen Patienten zu Völlegefühl und Appetitlosigkeit.

- Depressionen bei Patienten, die unter zunehmender Immobilität, Dyspnoe und Behinderung durch die Erkrankung leiden, sind häufig und führen ebenfalls zu verminderter Nahrungsaufnahme.

\section{Reduzierte Absorption}

Malabsorption spielt bei Patienten mit COPD keine wesentliche Rolle [7].

\section{Erhöhter Energieverbrauch}

Eine der Ursachen für einen höheren Ruhe-Energieverbrauch bei respiratorisch eingeschränkten Patienten ist die erhöhte Atemarbeit. Bei Gesunden werden in Ruhe ca. 2-3\% der Gesamtenergie für die Atmung benötigt. Bei Patienten mit COPD wird alleine aufgrund der verminderten Elastizität des Thorax und der mechanisch ungünstigeren Ausgangslage bei Überblähung mehr Energie für die Atmung benötigt [10]. Ob bei Patienten mit Lungenerkrankungen in einer stabilen Phase ein hypermetabolischer Zustand vorliegt, wird kontrovers diskutiert. Sicher ist dies aber der Fall bei Infekten, die bei diesen Patienten gehäuft auftreten. Es gibt jedoch Hinweise darauf, dass auch bei stabilen COPD-Patienten einige inflammatorische Zytokine erhöht sind und diese für einen Hypermetabolismus verantwortlich sind. Insbesondere TNF- $\alpha$ wurde untersucht, das im Sputum und Plasma von Patienten mit COPD als erhöht beschrieben wird. Hierbei fand sich, dass Patienten mit Untergewicht und/oder Gewichtsverlust höhere Werte von TNF- $\alpha$ zeigten als gewichtsstabile. Für die löslichen TNF-Rezeptoren (sTNF-R 55 und sTNF-R-75) liegen unterschiedliche Ergebnisse vor [6,9,31]. Unklar bleibt allerdings, ob der beobachtete höhere TNF- $\alpha$-Spiegel bei Patienten mit Untergewicht Ursache für den Gewichtsverlust ist, oder ob er durch den Gewichtsverlust verursacht wird. Eine Studie, die 
zeigte, dass anorektische Patienten einen hohen TNF-Spiegel haben und nach Nahrungszufuhr dieser Spiegel sank, sprechen für letzteres [46]. Aber auch Hypoxie führt zu einer Aktivierung des TNF- $\alpha$-Systems und könnte bei COPD-Patienten eine Rolle spielen [45]. Letztlich ist noch nicht völlig klar, ob TNF- $\alpha$ und andere inflammatorische Zytokine veranwortlich für den Gewichtverlust sind oder ob dies sekundäre Phanomene sind.

Auch in der Behandlung der Lungenerkrankungen häufig eingesetzte Medikamente wie Theophyllin und $\beta_{2}$-Sympathomimetika führen zu einer Erhöhung des Grundumsatzes. Kortikosteroide in höheren Dosierungen (>60 mg/d) verursachen eine Reduktion der Atemmuskelstärke [51]. Nicht ganz so klar ist der Effekt einer niedrigen Steroid-Dosis ( $<20 \mathrm{mg} / \mathrm{d})$. Die meisten Studien deuten aber auch bei den niedrigeren Dosen auf einen negativen Effekt auf die Atemmuskulatur bei Patienten mit fortgeschrittenen Lungenerkrankungen hin $[8,49]$.

Es gibt verschiedene Möglichkeiten, den Ruhe-Energiebedarf (Grundumsatz) zu berechnen. Die bekannteste Formel ist die Harris-Benedict-Formel [22], die für Patienten mit COPD modifiziert wird [29]

† $655+(9,56 \times \mathrm{KG}[\mathrm{kg}]+1,85 \times$ Größe $[\mathrm{cm}])-$

$(4,68 \times$ Alter [Jahre] $)$

^ $66,5+(13,7 \times$ Gewicht $[\mathrm{kg}]+5 \times$ Größe $[\mathrm{cm}])-$

$(6,78 \times$ Alter [Jahre] $)$

modifiziert für COPD

o $515+(14,1 \times$ Gewicht $[\mathrm{kg}]+1,85 \times$ Größe $[\mathrm{cm}])-$

$(4,68 \times$ Alter [Jahre] $)$

ô $952+(11,5 \times$ Gewicht $[\mathrm{kg}]+5 \times$ Größe $[\mathrm{cm}])-$

$(6,78 \times$ Alter [Jahre] $)$

\section{Veränderter Metabolismus}

In den letzten Jahren ist zunehmend darüber Erkenntnis gewonnen worden, dass der Metabolismus bei Patienten mit COPD gegenüber Gesunden verändert ist. Hierbei ist auch ein Unterschied zwischen Patienten mit Emphysem und chronischer Bronchitis zu beobachten. Diese Veränderungen wurden insbesondere durch Analysen von Muskeln gewonnen. So ist beschrieben, dass die Zusammensetzung der Muskelfasertypen sich zwischen Gesunden und COPD-Patienten unterscheiden [35]. Das Überwiegen von anaeroben Typ-1-Muskelfasern bei COPD-Patienten könnte mitverantwortlich sein für die frühere Ermüdung der Patienten, weil diese Muskeln ATP weniger effizient produzieren und die Produktion von Laktat höher ist. Auch die Aktivität der oxidativen Enzyme im Muskel von COPD-Patienten wurde als deutlich erniedrigt beschrieben [27].

In mehreren Studien wurde nachgewiesen, dass die Aminosäurezusammensetzung sowohl im Blut als auch im Muskel bei Patienten mit COPD verändert ist $[23,32]$. Engelen konnte kürzlich zeigen, dass auch bei COPD Patienten ohne Hypoxämie Veränderungen der Aminosäurezusammensetzung in Plasma und Muskel (M. quadriceps) zu finden sind [17]. Insbesondere fanden sich bei COPD-Patienten niedrigere Spiegel der verzweigtkettigen Aminosäuren, besonders Leucin. Interessanterweise bestand auch ein Unterschied zwischen COPD-Patienten mit und ohne Emphysem. Bei den Emphysematikern waren die Unterschiede zu den Gesunden noch deutlicher. Erhöhte Glutaminspiegel wurden bei den COPD-Patienten ohne Emphysem beobachtet, während sie bei Patienten mit Emphysem eher erniedrigt waren $[13,32]$. Sie sprechen für einen erhöhten Muskel-Protein-Turnover. Inwieweit die ebenfalls beobachtete Hyperinsulinämie bei den Patienten dafür mitverantwortlich ist, ist derzeit noch ungeklärt. Auch die Untersuchung des Protein-Breakdowns und der Proteinsynthese mittels Infusion von Phe- und Tyr-gelabelten Tracern zeigte, dass dieser bei COPD-Patienten gegenüber Gesunden erhöht war [15].

Interessanterweise verändert sich der Aminosäuremetabolismus nach Belastung. Engelen untersuchte die Zusammensetzung der Aminosäuren bei Gesunden und COPD-Patienten vor und nach Belastung [16]. Hierbei zeigte sich, dass bei COPD-Patienten die Konzentration fast aller Aminosäuren im Muskel nach Belastung erniedrigt war, im Plasma dagegen erhöht, ein weiterer Hinweis auf einen erhöhten Umsatz und Ausstrom von Aminosäuren aus dem Muskel bei COPDPatienten.

Welche Rolle die Erniedrigung der Fettmasse bei COPDPatienten spielt, ist derzeit noch unklar. Fett ist nicht nur Energiereservoir, sondern spielt auch eine Rolle bei unterschiedlichen Stoffwechselprozessen. Auch die Rolle von Leptin bei COPD wird kontrovers diskutiert. Leptin wurde vor wenigen Jahren entdeckt und unzählige Studien bei Überund Untergewicht publiziert. Tabatake und Schols fanden einen erniedrigten Leptin-Spiegel bei COPD-Patienten, der mit dem BMI korrelierte $[36,44]$. Schols konnte zeigen, dass der Spiegel besonders bei Emphysempatienten erniedrigt war. Die Korrelation zwischen Zytokinen und Leptin war in beiden Studien nicht eindeutig, lediglich für sTNF-R55 fand sich bei Emphysematikern eine Korrelation zum Leptin-Spiegel. Welche Rolle Leptin bei COPD spielt und wie es reguliert wird, bedarf weiterer Studien.

\section{Ernährungs-Intervention bei COPD}

Inzwischen liegen einige Studien vor, die zeigen konnten, dass eine Verbesserung des Ernährungszustandes bei untergewichtigen COPD-Patienten zu einer Kräftigung der Atemmuskulatur und besseren Funktionsparametern führen kann [11,52, 54]. Einige Autoren berichten aber auch, dass trotz einer Gewichtssteigerung kein Effekt auf die Atemmuskulatur zu beobachten war $[26,33]$. In den meisten Studien kam aber auch zum Ausdruck, dass die gesteigerte Kalorienzufuhr bei vielen COPD-Patienten nicht unproblematisch war. In erster Linie sind die oben aufgeführten Gründe für eine geringe Nahrungsaufnahme verantwortlich. Obwohl Rehabilitationsmaßnahmen kombiniert mit Ernährungsberatung und -intensivierung zusammen mit körperlichem Training die sinnvollste Maßnahme sind, gibt es derzeit keine aussagekräftigen Studien, in welchem Ausmaß sich hierdurch Lungenfunktion und Belastbarkeit steigern lassen. So zeigt eine bei älteren Patienten durchgeführte Studie, bei der Ernährungstherapie und körperliches Training mit alleiniger Ernährungstherapie verglichen wurde, bei Kombination einen positiven Effekt, der alleine nicht erreicht wurde [20].

Eine kürzlich erschienene Studie untersuchte die Akut-Effekte einer Supplementnahrung bei COPD-Patienten [48]. Die $\mathrm{Zu}-$ fuhr von $1048 \mathrm{~kJ}$ und $2092 \mathrm{~kJ}$ zeigte keinen wesentlichen 
messbaren negativen Effekt auf die Ventilation der Patienten, die niedrigere Zufuhr zeigte aber eine bessere metabolische und ventilatorische Antwort und ein geringeres Völlegefühl. Interessanterweise fand sich eine deutlichere Zunahme der Dyspnoe nach der fettreichen Nahrung als nach der kohlehydratreichen.

Es ist bis heute unklar, ob eine Nahrungssupplementierung das Überleben dieser Patienten verbessert, was gerade im Hinblick auf Kosten und dem Problem, das Gewicht zu halten, eine entscheidende Frage für weitere Langzeit-Studien sein muss. Eine Meta-Analyse aus 272 Publikationen fand kürzlich, dass nur neun randomisierte kontrollierte Studien zur Ernährungssupplementierung bei COPD-Patienten vorliegen. Der Benefit einer solchen Therapie war minimal und es konnte kein Effekt auf die Lungenfunktion und Belastbarkeit gezeigt werden [18]. Zudem zeigte die Analyse, dass die Gabe von Wachstumshormonen nicht effektiv ist.

Ein positiver Effekt auf die Lungenfunktion wurde bei der Einnahme von Zitrusfrüchten, Obst sowie dunkelgrünem Blatt und tiefgelbem bis orangenem Gemüse beschrieben $[43,47]$. In einer retrospektiven Auswertung der Essgewohnheiten von Rauchern fand Watson, dass die moderate Einnahme von Obst und Gemüse, insbesondere Apfel, einen signifikanten protektiven Effekt hat und das Risiko für die Entwicklung einer COPD bei Rauchern verringert. Eine der wenigen Studien, die den alten Spruch „An apple a day keeps the doctor away“ wissenschaftlich zu belegen versuchte [50].

Neben einer Ernährungsberatung gehören zur adäquaten Behandlung von COPD Patienten:

- Optimierung der Lungenfunktion

- regelmäßiges körperliches Training, anfänglich unter Anleitung, dann selbständig

- Verbesserung der Sauerstoffsättigung durch Sauerstoffgabe

- konsequente Behandlung von Infekten und deren Vorbeugung durch Impfungen (Grippe-Impfung und Pneumokokken-Impfung)

\section{Allgemeine Empfehlungen zur Ernährung bei Patienten mit COPD}

Adäquate Kalorienzufuhr (evtl. mit Zusatznahrung)

häufige kleine Mahlzeiten

Anraten von Mahlzeiten, die wenig Vorbereitung benötigen (z.B. Mikrowellen-Fertigmahlzeiten)

Ruhezeiten vor und nach den Mahlzeiten

Zufuhr von frischem Obst und Gemüse

\section{Schlussfolgerung}

In den letzten Jahren ist zunehmend die Erkenntnis gewonnen worden, dass bei der COPD viele Faktoren auf das Problem Unterernährung Einfluss haben. Hierbei wurde klar, dass eine komplexe Veränderung des Metabolismus bei COPD-Patienten vorliegt, die durch eine reine Ernährungstherapie wenig $\mathrm{zu}$ beeinflussen ist. Dies ist mit großer Wahrscheinlichkeit auch die Ursache für die geringen Erfolge einer Intervention durch Ernährung. Unser Blickfeld bezüglich der Therapie muss sich dahin richten, dass wir bei der COPD nicht eine lokale Erkrankung des Bronchialbaumes haben, sondern eher eine systemische Erkrankung, die von der Lunge ausgeht und dort die Hauptsymptome verursacht, aber auch andere Systeme mit einbezieht.

Ziel weiterer Studien muss es daher sein, die Muskelfunktion und die metabolischen Veränderungen bei COPD-Patienten noch besser zu verstehen, um dann hierauf Einfluss nehmen zu können. Zukünftige Therapien sollten sich daher nicht nur auf die Behandlung der bronchialen Inflammation und Bronchodilatation beschränken, sondern darauf ausgerichtet sein, den veränderten Metabolismus bei COPD-Patienten zu beeinflussen, um so die Belastbarkeit der Patienten und vielleicht auch die Prognose zu verbessern.

\section{Literatur}

${ }^{1}$ Arora NS, Rochester DF. Effect of body weight and muscularity on human diaphragm muscle mass, thickness, and area. J Appl Physiol 1982; 52 (1): $64-70$

${ }^{2}$ Baarends EM, Schols AM, Mostert R et al. Peak exercise response in relation to tissue depletion in patients with chronic obstructive pulmonary disease. Eur Respir J 1997; 10 (12): 2807 - 2813

${ }^{3}$ Bernard S, LeBlanc P, Whittom $F$ et al. Peripheral muscle weakness in patients with chronic obstructive pulmonary disease. Am J Respir Crit Care Med 1998; 158 (2): 629-634

${ }^{4}$ Braun SR, Keim NL, Dixon RM et al. The prevalence and determinants of nutritional changes in chronic obstructive pulmonary disease. Chest 1984; 86 (4): $558-563$

${ }^{5}$ Chailleux E, Fauroux B, Binet F et al. Predictors of survival in patients receiving domiciliary oxygen therapy or mechanical ventilation. A 10-year analysis of ANTADIR observatory. Chest 1996; 109 (3): 741 - 749

${ }^{6}$ Creutzberg EC, Wouters EF, Vanderhoven-Augustin IM, Dentener MA, Schols AM. Disturbances in leptin metabolism are related to energy imbalance during acute exacerbations of chronic obstructive lung disease. Am J Respir Crit Care Med 2000; 162 (4): $1239-1245$

7 d'A Semple P, Watson WS, Beastall GH et al. Diet, absorption and hormone studies in relation to body weight in obstructive airways disease. Thorax 1979; 34 (6): $783-788$

${ }^{8}$ Decramer $M$, Lacquet LM, Fagard $\mathrm{R}$ et al. Corticosteroids contribute to muscle weakness in chronic airflow obstruction. Am J Respir Crit Care Med 1994; 150 (1): 11 - 16

${ }^{9}$ Di Francia M, Barbier D, Mege JL, Orehek J. Tumor necrosis factor-alpha levels and weight loss in chronic obstructive pulmonary disease. Am J Respir Crit Care Med 1994; 150: 1453 1355

${ }^{10}$ Donahoe M, Rogers RM, Wilson DO et al. Oxygen consumption of the respiratory muscles in normal and in malnourished patients with chronic obstructive pulmonary disease. Am Rev Respir Dis 1989; 140 (2): 385-391

${ }^{11}$ Efthimiou J, Fleming J, Gomes $C$ et al. The effect of supplementary oral nutrition in poorly nourished patients with chronic obstructive pulmonary disease. Am Rev Respir Dis 1988; 137 (5): 1075-1082

${ }^{12}$ Engelen MP, Schols AM, Baken WC et al. Nutritional depletion in relation to respiratory and peripheral skeletal muscle function in out-patients with COPD. Eur Respir J 1994; 7 (10): 1793 - 1797

${ }^{13}$ Engelen MP, Schols AM, Does JD et al. Altered glutamate metabolism is associated with reduced muscle glutathione levels in patients with emphysema. Am J Respir Crit Care Med Jan 2000; 161 (1): 98 - 103

${ }^{14}$ Engelen MP, Schols AM, Lamers RJ et al. Different patterns of chronic tissue wasting among patients with chronic obstructive pulmonary disease. Clinical Nutrition 1999; 18 (5): 275-280 
${ }^{15}$ Engelen MPKJ, Deutz NEP, Wouters EFM et al. Enhanced levels of whole-body protein turnover in patients with chronic obstructive pulmonary disease. Am J Respir Crit Care Med 2000; 162: $1488-1492$

${ }^{16}$ Engelen MPKJ, Wouters EFM, Deutz NEP et al. Effects of exercise on amino acid metabolism in patients with chronic obstructive pulmonary disease. Am J Respir Crit Care Med 2001; 163: 859864

17 Engelen MPKJ, Wouters EFM, Deutz NEP et al. Factors contributing to alterations in skeletal muscle and plasma amino acid profiles in patients with chronic obstructive pulmonary disease. Am J Clin Nutr 2000; 72: 1480-1487

${ }^{18}$ Ferreira IM, Brooks D, Lacasse Y et al. Nutritional support for individuals with COPD. A meta-analysis. Chest 2000; 117: 672 678

${ }^{19}$ Fiaccadori E, Del Canale S, Coffrini E et al. Hypercapnic-hypoxemic chronic obstructive pulmonary disease (COPD): influence of severity of COPD on nutritional status. Am J Clin Nutr 1988; 48 (3): $680-685$

${ }^{20}$ Fiatarone MA, O\&Neill EF, Ryan ND et al. Exercise training and nutritional supplementation for physical frailty in very elderly people. N Engl J Med 1994; 330 (25): 1769-1775

${ }^{21}$ Gray-Donald K, Gibbons L, Shapiro SH et al. Effect of nutritional status on exercise performance in patients with chronic obstructive pulmonary disease. Am Rev Respir Dis 1989; 140 (6): 1544 1548

${ }^{22}$ Harris JA, Benedict FG. Standard basal metabolism constants for physiologists and clinicians: a biometric study of basal metabolism in man; Lippincott: in Anonymous Philadelphia, 1919

${ }^{23}$ Hofford JM, Milakofsky L, Vogel WH et al. The nutritional status in advanced emphysema associated with chronic bronchitis. A study of amino acid and catecholamine levels. Am Rev Respir Dis 1990; 141: $902-908$

${ }^{24}$ Hunter AM, Carey MA, Larsh HW. The nutritional status of patients with chronic obstructive pulmonary disease. Am Rev Respir Dis 1981; 124 (4): 376-381

${ }^{25}$ Kelsen SG. The effects of undernutrition on the respiratory muscles. Clin Chest Med 1986; 7 (1): $101-110$

${ }^{26}$ Knowles JB, Fairbarn MS, Wiggs BJ et al. Dietary supplementation and respiratory muscle performance in patients with COPD. Chest 1988; 93: 977-983

${ }^{27}$ Maltais F, Simard AA, Simard C et al. Oxidative capacity of the skeletal muscle and lactic acid kinetics during exercise in normal subjects and in patients with COPD. Am J Respir Crit Care Med 1996; 153: 288-293

${ }^{28}$ Martin TR, Altman LC, Alvares OF. The effects of severe proteincalorie malnutrition on bacterial defense mechanisms in the rat lung. Am Rev Respir Dis 1983; 128 (6): 1013-1019

${ }^{29}$ Moore JA, Angelillo VA. Equations for the prediction of resting energy expenditure in chronic obstructive lunge disease. Chest 1988; 94: $1260-1263$

${ }^{30}$ Mostert R, Goris A, Weling-Scheepers C et al. Tissue depletion and health related quality of life in patients with chronic obstructive pulmonary disease. Respir Med Sep 2000; 94 (9): $859-867$

${ }^{31}$ Nguyen LT, Bedu M, Caillaud D, Beaufrere B, Beaujon G, Vasson $\mathrm{M}$, Coudert J, Ritz P. Increased resting energy expenditure is related to plasma TNF-alpha concentration in stable COPD patients. Nutrition 1999; 18 (5): 255-257

32 Pouw EM, Schols AM, Deutz NE et al. Plasma and muscle amino acid levels in relation to resting energy expenditure and inflammation in stable chronic obstructive pulmonary disease. Am J Respir Crit Care Med 1998; 158: 797-801

${ }^{33}$ Rogers RM, Donahoe M, Costantino J. Physiologic effects of oral supplemental feeding in malnourished patients with chronic obstructive pulmonary disease. Am Rev Respir Dis 1992; 146 : $1511-1517$

${ }^{34}$ Sahebjami H, Doers JT, Render ML et al. Anthropometric and pulmonary function test profiles of outpatients with stable chronic obstructive pulmonary disease. Am J Med 1993; 94 (5): $469-474$

${ }^{35}$ Satta A, Migliori GB, Spanevello A et al. Fibre types in skeletal muscles of chronic obstructive pulmonary disease patients related to respiratory function and exercise tolerance. Eur Respir J 1997; 10: 2853 - 2860

${ }^{36}$ Schols AM, Creutzberg EC, Buurman WA et al. Plasma leptin is related to proinflammatory status and dietary intake in patients with chronic obstructive pulmonary disease. Am J Respir Crit Care Med 1999; 160 (4): 1220-1226

${ }^{37}$ Schols AM, Mostert R, Soeters PB et al. Body composition and exercise performance in patients with chronic obstructive pulmonary disease. Thorax 1991; 46: 695-699

${ }^{38}$ Schols AM, Soeters PB, Dingemans AM et al. Prevalence and characteristics of nutritional depletion in patients with stable COPD eligible for pulmonary rehabilitation. Am Rev Respir Dis 1993; 147 (5): $1151-1156$

${ }^{39}$ Shennib H, Chiu RC, Mulder DS et al. Depression and delayed recovery of alveolar macrophage function during starvation and refeeding. Surg Gynecol Obstet 1984; 158 (6): 535-540

${ }^{40}$ Shoup R, Dalsky G, Warner S et al. Body composition and healthrelated quality of life in patients with obstructive airways disease. Eur Respir J 1997; 10 (7): 1576-1580

${ }^{41}$ Skerrett SJ, Henderson WR, Martin TR. Alveolar macrophage function in rats with severe protein calorie malnutrition. Arachidonic acid metabolism, cytokine release, and antimicrobial activity. J Immunol 1990; 144 (3): $1052-1061$

${ }^{42}$ Smeenk FWJM, Creutzberg EC, Fokkens B et al. COPD and seretide: a multi-center intervention and characterisation (COSMIC) study: prevalence of nutritional depletion in patients with COPD stratified by disease stage. Am J Respir Crit Care Med 2001; 163: A 502

${ }^{43}$ Tabak C, Smit HA, Rasanen L et al. Dietary factors and pulmonary function: a cross sectional study in middle aged men from three European countries. Thorax 1999; 54: 1021 - 1026

${ }^{44}$ Takabatake N, Nakamura H, Abe S et al. Circulating leptin in patients with chronic obstructive pulmonary disease. Am J Respir Crit Care Med 1999; 159 (4 Pt 1): 1215-1219

${ }^{45}$ Takabatake N, Nakamura H, Abe S, Inoue H, Hino T, Saito H, Yuki $\mathrm{H}$, Kato $\mathrm{S}$, Tomoike $\mathrm{H}$. The relationship between chronic hypoxemia and activation of the tumor necrosis factor-alpha system in patients with chronic obstructive pulmonary disease. Am J Respir Crit Care Med 2001; 161 (4): 1179-1184

${ }^{46}$ Vaismann N, Hahn T. Tumor necrosis factor-alpha and anorexia cause or effect? Metabolism 1991; 40 (7): 720-723

${ }^{47}$ Van Duyn MA, Pivonka E. Overview of the health benefits of fruit and vegetable consumption for the dietetics professional: selected literature. J Am Diet Assoc Dec 2000; 100 (12): 1511 - 1521

${ }^{48}$ Vermeeren MAP, Wouters EF, Nelissen LH, van Lier A, Hofmann $Z$, Schols AM. Acute effects of different nutritional supplements on symptoms and functional capacity in patients with chronic obstructive pulmonary disease. Am J Clin Nutr 2001; 73: 295301

${ }^{49}$ Wang YM, Zintel T, Vasquez A et al. Corticosteroid therapy and respiratory muscle function in humans. Am Rev Respir Dis 1991; 144 (1): $108-112$

${ }^{50}$ Watson L, Margetts BM, Howarth P, Dorward MA, Thompson R, Little PS. Do dietary factors protect against chronic pulmonary disease: a case control study. Am J Respir Crit Med 2001; 163: A40 
${ }^{51}$ Weiner P, Azgad Y, Weiner M. The effect of corticosteroid on inspiratory muscle performance in humans. Chest 1993; 104 (6): $1788-1791$

52 Whittaker JS, Ryan CF, Buckley PA et al. The effects of refeeding on peripheral and respiratory muscle function in malnourished chronic obstructive pulmonary disease patients. Am Rev Respir Dis 1990; 142 (2): 283-288

${ }^{53}$ Wilson DO, Rogers RM, Sanders MH et al. Nutritional intervention in malnourished patients with emphysema. Am Rev Respir Dis 1986; 134: 672-677

${ }^{54}$ Wilson DO, Rogers RM, Wright EC et al. Body weight in chronic obstructive pulmonary disease. The national institutes of health intermittent positive-pressure breathing trial. Am Rev Respir Dis 1989; 139 (6): 1435 - 1438

${ }^{55}$ Yoshikawa M, Yoneda T, Kobayashi A et al. Body composition analysis by dual energy X-ray absorptiometry and exercise performance in underweight patients with COPD. Chest 1999; 115: $371-375$

${ }^{56} \mathrm{Zu}$ Wallack RL, Shoup R, Warner S et al. Alterations in total and regional body composition in patients with moderate to severe obstructive lung disease. Monaldi Arch Chest Dis 1996; 51: $507-509$

\section{PD Dr. J. Bargon}

Leiter der Abteilung Pneumologie/Allergologie

St. Elisabethenkrankenhaus

Ginnheimer Straße 3

60487 Frankfurt am Main

E-mail: bargon@em.uni-frankfurt.de 